\title{
Invasive squamous cell carcinoma of the vulva clinically mimicking a diabetic ulcer: A potential diagnostic pitfall
}

\author{
Jessian L Munoz ${ }^{1}$, Christopher G. Przybycin ${ }^{2}$ and Oluwatosin Jaiyeoba Goje ${ }^{1 *}$ \\ ${ }^{1} \mathrm{OB} / \mathrm{GYN}$ and Women's Health Institute, Cleveland Clinic, 9500 Euclid Avenue A81, Cleveland, OH, USA. \\ ${ }^{2}$ Robert J. Tomsich Pathology and Laboratory Medicine Institute, Cleveland Clinic, 9500 Euclid Avenue L25, Cleveland, OH, USA
}

\begin{abstract}
Background: Vulvar cancer is an uncommon disease with significant morbidity and mortality. Chronic conditions such as hypertension and diabetes may overshadow and can delay the timely diagnosis of vulvar cancer. While chronic and poorly managed diabetes may present with non-healing ulcers, vulvar cancer may also present as a persistent vulvar lesion or ulcer of the lower genital tract.

Case: Patient is a 49 year-old woman who presented with a persistent non-healing vulvar ulcer thought to be secondary to poorly controlled diabetes. This was later uncovered to be her initial presentation of vulvar carcinoma.

Conclusion: Our report presents a case where the diagnosis of vulvar squamous cell carcinoma required high clinical suspicion and ultimately biopsy of the questionable lesion, which is not routinely performed on ulcers secondary to chronic conditions like diabetes due to fear of poor wound healing.
\end{abstract}

\section{Introduction}

Vulvar cancer is an uncommon diagnosis representing less than $0.6 \%$ of cancer diagnoses in the United States [1]. The 5-year survival rate of the vulvar cancer remains approximately $70 \%$ [2]. $80 \%$ of vulvar cancer diagnoses are in women $>50$ years old. Given this ageproportionate increase in vulvar cancer prevalence, the diagnosis may be complicated by a number of chronic disorders such as lichen sclerosis and planus as well as systemic manifestations of diseases such as hypertension/peripheral arterial disease and diabetes mellitus [3]. Yet, given the low incidence of vulvar cancer and epidemiologic data, diabetes and hypertension have not been shown to conclusively be risk factors of this malignancy [4]. We present a case of 49-year-old woman who initially presented with a non-healing ulcer attributed to her poorly controlled diabetes, yet this delayed her diagnosis and treatment of stage IIIC metastatic vulvar squamous cell carcinoma.

\section{Case report}

A 49-year-old G2P2 presented to the Cleveland Clinic Vulvovaginal health clinic after assessment by 2 gynecologists for an ulcerated right vulvar lesion. Of note, the patient had a BMI of 44 and a known history of poorly controlled type 2 diabetes mellitus, HgbA1C 9.5\%. The lesion in question was biopsied a year prior to presentation at another medical institution and pathology reported as squamous hyperplasia without dysplasia which was treated with clobetasol $0.05 \%$ for possible lichen sclerosus.

A year after the diagnosis of squamous hyperplasia without dysplasia, she presented to another gynecologist, and the lesion remained ulcerated and non-healing. Patient reported a burning sensation and skin sloughing. Examination of the vulva revealed tenderness and erythema, a right-sided $5 \mathrm{~cm}$ depression on right labia majus with heaped edges suggestive of ulceration not secondary to iatrogenic biopsy, additional smaller satellite ulceration and bleeding after gentle manipulation was noted (Figure 1). At that time, decision was made to optimize glycemic control in an effort to improve wound healing. Patient was referred to an Endocrinologist. In addition, a persistent yeast infection was noted and the patient was referred to the Cleveland Clinic Vulvo-vaginal health clinic for management of persistent vulvo-vaginal candidiasis.

At the Cleveland Clinic, a right vulvar lesion was noted. There was erythema of the vulva and the right side had a shallow ulcer of $0.3 \mathrm{~cm}$

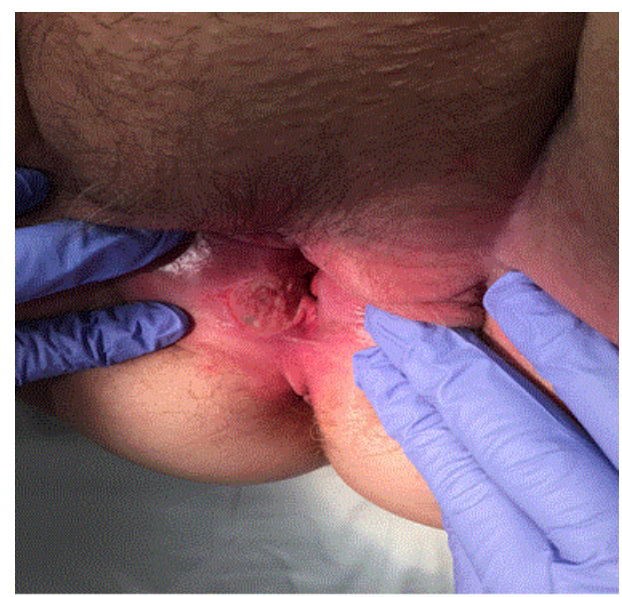

Figure 1. Erythematous vulvar lesion. Shallow tender, non-healing ulcer of $0.3 \mathrm{~cm}$ in depth, involving the right vestibule, extending past the hymenal ring, $6 \mathrm{~cm}$ in length by 3 $\mathrm{cm}$ in height. The ulcer had raised firm edges, a grey fibrinous base and bled on contact.

Correspondence to: Oluwatosin Jaiyeoba Goje, OB/GYN and Women's Health Institute, Cleveland Clinic, 9500 Euclid Avenue A81, Cleveland, OH, USA, Tel: (216) 444-5857; E-mail: gojeoo@ccf.org

Received: May 02, 2017; Accepted: May 15, 2017; Published: May 17, 2017 
in depth, involving the right vestibule, extending past the hymenal ring, $6 \mathrm{~cm}$ in length by $3 \mathrm{~cm}$ in height. The ulcer had raised firm edges, a grey fibrinous base and bleed on contact. Decision was made to perform punch biopsies of the lesion incorporating the edges and the base into biopsied specimen. Pap smear and saline microscopy was performed at the same time. Saline microscopy with addition of $10 \%$ potassium hydroxide showed pseudohyphae consistent with vulvovaginal candidiasis and the Pap smear was reported as ASCUS HPV negative. The vulvar biopsy contained an ulcerated invasive poorly differentiated carcinoma consisting of an irregularly infiltrative population of epithelioid cells with abundant dense eosinophilic cytoplasm and pleomorphic nuclei with irregular contours, open chromatin, and prominent nucleoli, growing in irregular cords and nests, morphologically consistent with a poorly differentiated squamous cell carcinoma. Immunohistochemical stains were performed to confirm the diagnosis and to exclude other malignancies (e.g. melanoma, epithelioid angiosarcoma). The tumor cells were positive for markers expressed in squamous cell carcinoma (p63, cytokeratin 5/6), while negative for S100 (a melanoma marker) and ERG (a vascular marker), confirming the morphologic impression (Figure 2). P16 was negative, suggesting a non-HPV associated carcinoma. Patient was referred to GYN Oncology for further management.

Patient underwent radical vulvectomy, distal urethrectomy, and bilateral groin node dissection. Surgical pathology demonstrated poorly differentiated squamous cell carcinoma with metastasis to the right groin nodes with extracapsular extension consistent with stage IIIc disease. She subsequently received radiation and Cisplatin chemotherapy with no residual disease at that time. Three months later, she was noted to recur with lung involvement.

\section{Discussion}

Timely diagnosis and treatment is essential for cancer therapy. As the general age of the population continues to climb with improved healthcare, cancer diagnoses will present along with known chronic conditions. Our particular case is one such example, in which vulvar squamous cell carcinoma clinically mimicked a diabetic ulcer.

Vulvar cancer is most commonly squamous cell carcinoma, but also includes melanoma,adenocarcinoma (of the Bartholin's gland),

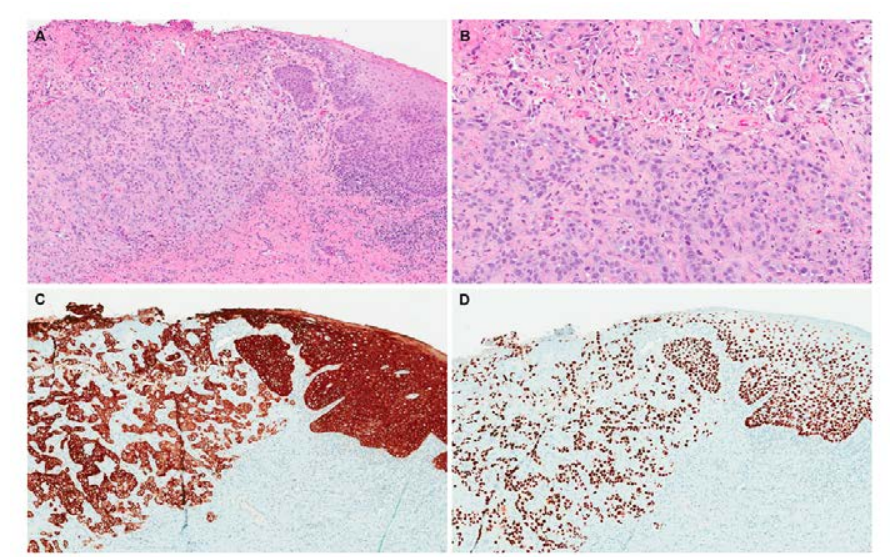

Figure 2. Microscopic analysis of lesion. A. Low power examination shows squamous mucosa with an ulcer and an underlying infiltrative population of tumor cells (H\&E, 40X magnification). B. At higher magnification, the cells are arranged in cords and trabeculae and contain large nuclei with prominent nucleoli and irregular nuclear contours (H\&E, 100X magnification). C, D. Immunohistochemical stains for high molecular weight cytokeratin (CK 5/6, figure 2C) and p63 (figure 2D) are diffusely and strongly positive, supporting a diagnosis of squamous cell carcinoma (40X magnification). and less commonly soft tissue sarcoma. Common signs and symptoms of vulvar cancer include itching, burning, bleeding and changes to color of the skin. Vulvar cancer may also present as non-healing ulcers, lumps or sores. Diagnosis is often delayed due to patient privacy and modesty. Yet, in this case the patient sought out care and the nonhealing ulcer was attributed to her known chronic disease of diabetes Interestingly, the non-healing ulcer may have been a manifestation of many conditions such as STIs (syphilis, chancroid, lymphogranuloma venereum and granuloma inguinale), a fungal infection, psoriasis or Behcet's disease among others. Ultimately, diagnosis requires careful examination of the vulva including the perineum, clitoris, urethra and Bartholin's glands. Any suspicious lesions should be biopsied.

In a study by Kouvaris et al., on univariate analysis, the authors identified possible prognostic factors for vulvar cancer [5]. Overall survival was impacted by advanced stage, ulceration, tumor size, obesity, smoking, diabetes and hypertension. Yet, these are prognostic factors and do not reflect risk factors or aid in the initial diagnosis.

Clinicians must have a high index of suspicion with any persistent and or non-healing ulcer. Single or multiple biopsies as the case may warrant should be performed or the patient can be referred to a specialist who can perform a biopsy and make a diagnosis. In the context of sufficient clinical suspicion of malignancy, he concerns about poor healing from a biopsy should not deter biopsy or delay management. In addition, patients can have more than one diagnosis; the presence of vulvo-vaginal candidiasis or lichen sclerosus does not eliminate malignancy especially when all treatment options for commonly known diseases have failed.

\section{Conclusion}

Vulvar cancer is a rare but significant and potentially lifethreatening disease for women. Chronic medical conditions may overshadow obvious signs of malignancy. Clinical judgment should be the cornerstone in diagnosing gynecologic conditions. Suspicious lesions should be sampled regardless of the general health condition of the patient. Ultimately, cancer diagnosis requires tissue-proven pathology.

\section{Conflict of interest}

The authors declare that there is no conflict of interests regarding the publication of this paper.

\section{References}

1. Suh DH, Kim M, Kim HJ (2016) Major clinical research advances in gynecologic cancer in 2015. J Gynecol Oncol 27:e53.

2. Mahner S, Prieske K, Grimm D, Trillsch F, Prieske S, et al. (2015) Systemic treatment of vulvar cancer. Expert Rev Anticancer Ther 15: 629-637.[Crossref]

3. Hill-Daniel J, Roett MA (2015) Genital Cancers in Women: Vulvar Cancer. FP Essent 438: 31-43.[Crossref]

4. Kouvaris JR, Kouloulias VE, Loghis CD, Balafouta EJ, Miliadou AC, et al. (2001) Minor prognostic factors in squamous cell vulvar carcinoma. Eur J Gynaecol Oncol 22: 305-308.[Crossref]

5. Kouvaris J, Kouloulias V, Loghis C (2001) Prognostic factors for survival in invasive squamous cell vulvar carcinoma: a univariate analysis. Gynecol Obstet Invest 4: 262-265.

Copyright: C2017 Munoz JL. This is an open-access article distributed under the terms of the Creative Commons Attribution License, which permits unrestricted use, distribution, and reproduction in any medium, provided the original author and source are credited. 